\title{
Dynamic power flow tool development for low voltage networks analysis with high penetration level of distributed generation
}

\author{
A. Milo ${ }^{1}$, A. Martinez ${ }^{1}$, M. Rodríguez ${ }^{2}$, A. Goikoetxea ${ }^{2}$ \\ ${ }^{1}$ Departement of Control Engineering \\ IKERLAN, Technological Research centre \\ $\mathrm{P}^{\mathrm{o}}$ J.M. Arizmendiarrieta, 2, 20500 - Mondragón - Arrasate (Spain) \\ Phone/Fax: +34 943712400, +34 943796944, e-mail: amilo@ikerlan.es \\ ${ }^{2}$ Faculty of Engineering, University of Mondragon \\ Loramendi 4, Aptdo 23, 20500 - Mondragón-Arrasate (Spain) \\ e-mail: mrodriguez@eps.muni.es, agoikoetxtea@eps.muni.es
}

\begin{abstract}
This article presents the development of a power flow tool to analyse the impact of the integration of distributed generation on low voltage distribution networks. The work has been implemented in MATLAB-SIMULINK environment. The developed tool can be used in radial or weakly meshed 3-phase networks with the presence of distributed generation. The voltage and power magnitudes of selected nodes can vary over time allowing a dynamic power flow analysis.
\end{abstract}

\section{Key words}

3-phase analysis, low voltage (LV) network, dynamic power flow, distributed generation (DG).

\section{Introduction}

The appropriate integration of DG units into distribution networks can help to improve their efficiency from the point of view of the network operator, the integration of DG in the distribution networks could mean [1]:

- Reduction of electric losses.

- Reduction of congestion in the transmission and distribution networks.

- Postponement of investment for upgrading or new network infrastructure.

- Improvement of service reliability, energy quality, and voltage regulation.

From the point of view of the owner of a DG unit, this could be an interesting investment thanks to the incentives and special rates applied to the generation of electricity under the special regime, based on generation technologies that use renewable energies, waste and cogeneration, as in Spain [2]. In the same way, DG is an interesting option to cover all or part of the demand in remote areas or with high peak demands.

To optimise these benefits it is necessary to carry out the integration of DG in a controlled and co-ordinated way.
Poorly planned integration could lead to operational problems due to the voltage operating limits or the thermal limits for conductors being exceeded.

With the perspective of significant penetration of distributed generation on LV networks some applications will require repeated and fast load flow solution that must be resolved as efficiently as possible [3]:

- Distribution automation.

- Generator voltage correction.

- Optimization of power system.

- Control of sudden increase in demand or generation.

The different types of generator (single-phase, 2-phase and 3-phase connections) present on the LV network with balanced and unbalanced loads will require a power flow method that takes this aspect into consideration to obtain accurate results.

In order to be able to analyse these scenarios a 3-phase dynamic power flow tool has been developed. The tool is able of carrying out a dynamic analysis of radial or weakly meshed networks with the presence of distributed generation.

This paper describes at first the dynamic power flow modelling and implementation. Next details of the implementation are presented. At the end simulations, results and conclusions are presented.

\section{Dynamic Power Flow}

The main part of developed tool corresponds to the power flow calculation algorithm. The algorithm is especially suitable for the analysis of distribution networks with a high $\mathrm{R} / \mathrm{X}$ ratio and a radial or weakly meshed structure; enabling the 3-phase analysis of the network to be carried out with reduced calculation times. 
The method used in the power flow algorithm is based on the developments presented in [4] [5] [6] [7] [8]. This method takes advantage of the radial configuration of the distribution networks to carry out progressive sweeps, forwards and backwards, until convergence in their calculations is achieved. Unlike the traditional methods, Newton-Raphson or Gauss-Seidel, there is no need to manipulate the network's admittance matrix presenting good levels of convergence and calculation speed. The main characteristic of this algorithm is its convergence speed which makes it especially suitable for use in real time calculations where in each iteration is necessary to carry out a power flow calculation.

The developed algorithm has been tested on a 200-node radial network, converging in three iterations in a time period of 0.03 seconds. The same network has been tested with the traditional Newton-Raphson algorithm developed in [9], with convergence in three iterations in a time period of 0.07 seconds.

\section{A. Components model}

The modelling specifications provide the description of power system components. The different components of the network model are described below.

Line model:

The generic model used to represent the distribution line connecting two buses is based on the $\pi$-model as shown in figure 1 [7].

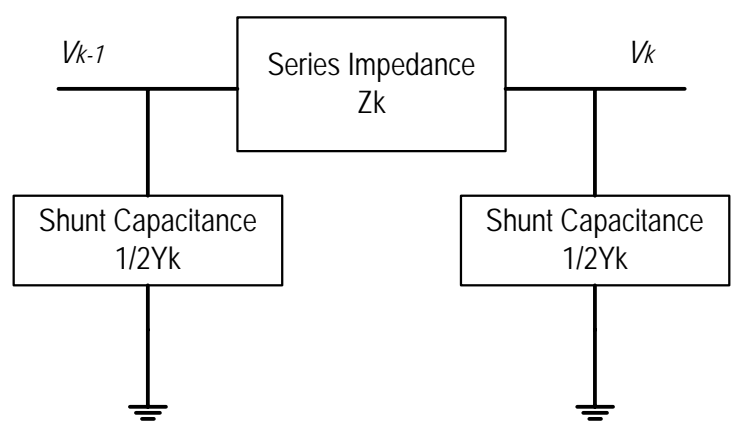

Fig. 1. Line model

Where $\mathrm{k}$ represents the entering node.

LV distribution networks have lengths where the effect of shunt capacitance can be discounted so that the $\pi$ line model can be simplified, bearing in mind the line's resistance and series inductance [10].

$$
Z_{k}=R_{k}+j X_{k}
$$

Load and DG model:

Loads can be modelled as constant-power, constantcurrent, constant impedance or a combination of these. In our model the constant-power is chosen where their known variables are consumed active and reactive power [11].

DG units are modelled as PV nodes where their known variables are voltage magnitude and injected real power, or as negative loads where their known variables are injected active and reactive power.

Some loads or DG units can be chosen like dynamic nodes where their known variables vary over time. To difference dynamic nodes from non dynamic nodes the loads and DG units are identified with an index.

PQ node: 1 PQ-dynamic node: 10

PV node: 2 PV-dynamic node: 20

Slack node: 3

\section{B. Implementation}

Implementation specifications indicate how to use the model to obtain the solutions. The description of the used method to solve the power flow is shown below.

\section{Backward-Forward Sweep:}

The forward sweep calculates the current from the end nodes to the slack node. This step uses equations (1) and (2).

$$
\begin{aligned}
& \tilde{I}_{L k}=\left(S_{L k} \cdot / V_{k}\right)^{*} \\
& I_{k}{ }^{\prime}=\tilde{I}_{L k}+\left(\sum_{j \in A_{k}} I_{j}\right)+I_{k+1}
\end{aligned}
$$

$I_{L k}:$ Current consumed by the load in node $\mathrm{k}$.

$I_{k}{ }^{\prime}$ : Current injected in $\mathrm{k}$ from the higher node $\mathrm{k}-1$.

$I_{j}:$ Current consumed by the nodes adjacent to node $\mathrm{k}$.

$I_{k+1}$ : Current consumed by the lower node $\mathrm{k}+1$.

Then, updating the slack node first, all the voltage values for all the nodes are updated using equation (3).

$$
\tilde{V}_{k}=V_{k-1}-Z_{k} I_{k}
$$

The sweep is repeated until the convergence criterion is achieved. The flow chart in figure 2 shows the operation sequence for the power flow calculation algorithm developed. 


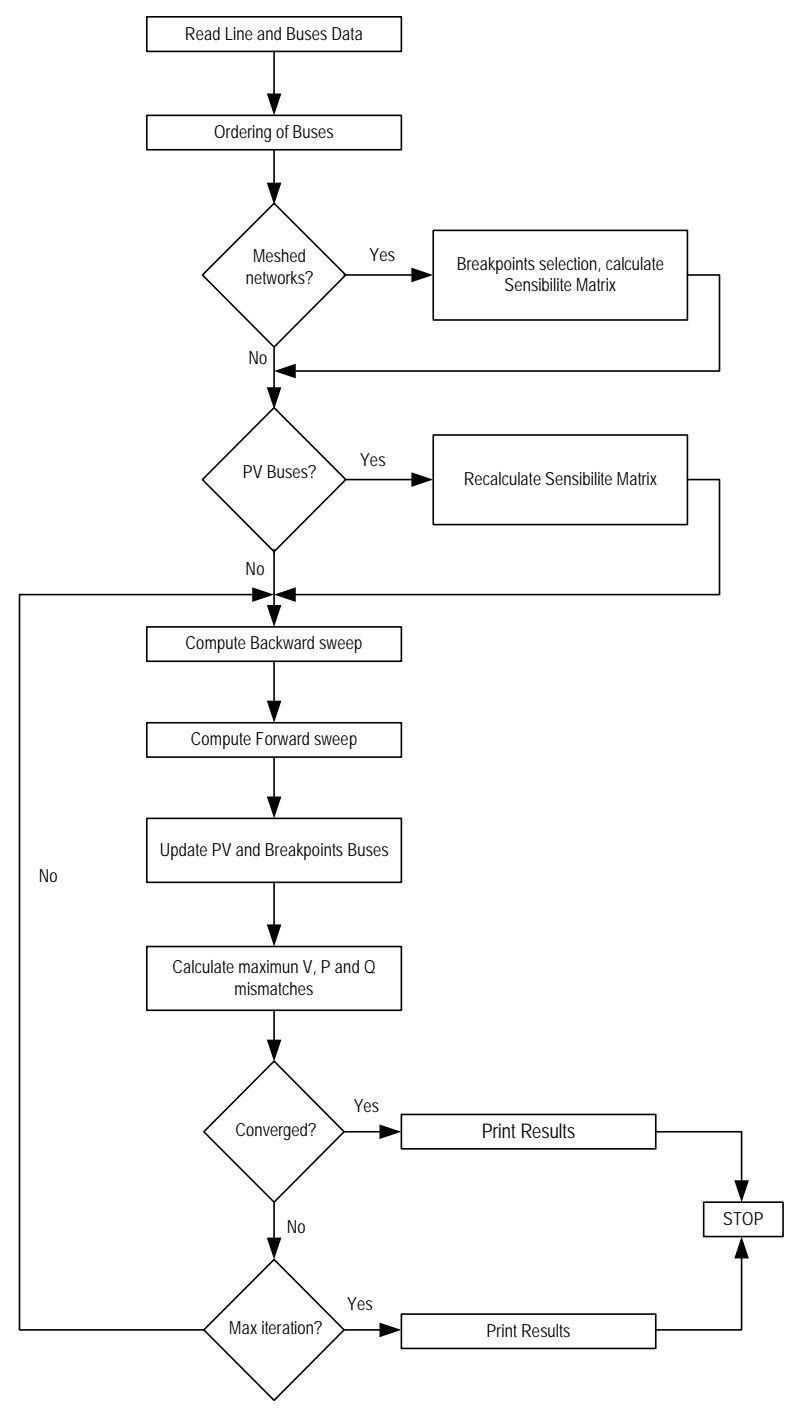

Fig. 2. Flow chart of power flow algorithm

Convergence:

The convergence criterion uses three inequations (4) (5) (6) which have to be complied with simultaneously:

$$
\begin{aligned}
& \text { tol }_{\Delta P} \geq \max \left(\Delta P_{1}, \Delta P_{2}, \ldots, \Delta P_{n+m-1}\right) \\
& \text { tol }_{\Delta Q} \geq \max \left(\Delta Q_{1}, \Delta Q_{2}, \ldots, \Delta Q_{n}\right) \\
& \text { tol }_{\Delta V} \geq \max \left(\Delta V_{1}, \Delta V_{2}, \ldots, \Delta V_{m}\right)
\end{aligned}
$$

Where:

$\mathrm{n}$ : Number of PQ type nodes.

m: Number of PV type nodes plus the slack node.

$\Delta P, \Delta Q, \Delta V$ : active and reactive power and voltage mismatches.

\section{Matlab-Simulink implementation}

The implemented power flow algorithm has been integrated in a SIMULINK MATLAB-function block.

The block's inputs are the known variables of the dynamic nodes established in the distribution network configuration file.

$P Q$ node inputs: $P a, P b, P c, Q a, Q b, Q c$.

PV node inputs: $P a, P b, P c, \vec{V}_{a}, \vec{V}_{b}, \vec{V}_{c}$.

The block's outputs are the voltages in each of the network's nodes.

Outputs : $\vec{V}_{a}, \vec{V}_{b}, \vec{V}_{c}$

Figure 3 shows the dynamic power flow calculation model developed in MATLAB-SIMULINK.

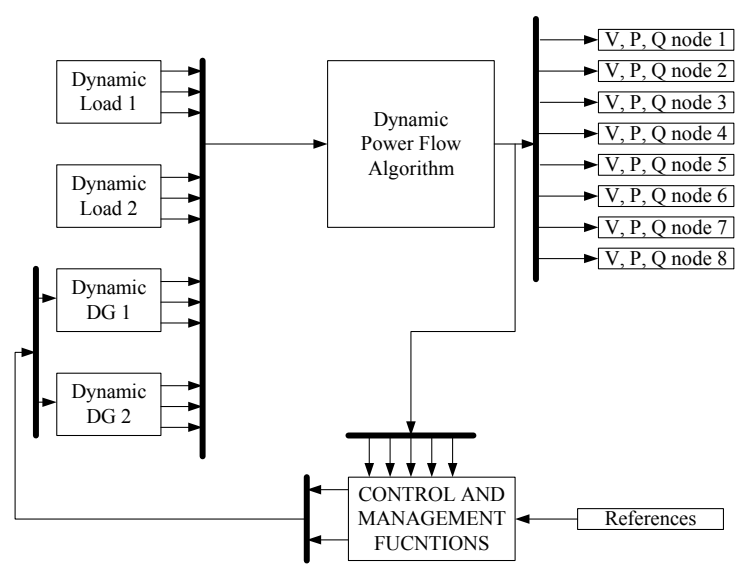

Fig. 3. Dynamic power flow model

\section{Simulation scenario}

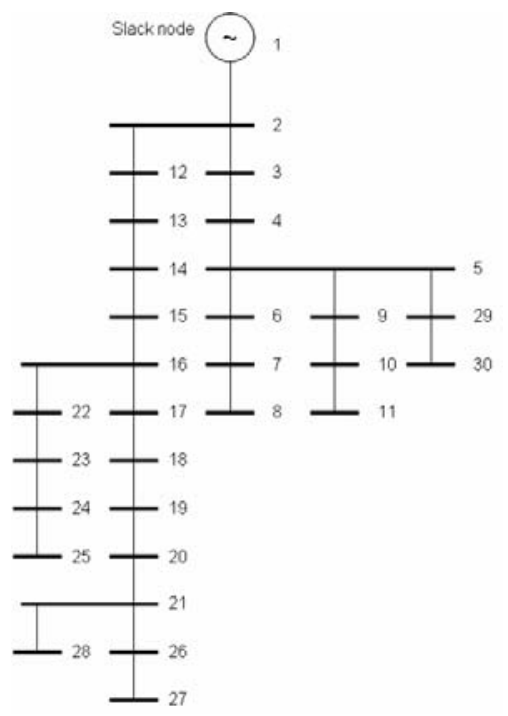

Fig. 4. Simulation scenario, Annexe 1[12] 
The tool has been tested on a 400/230 volts 3-phase grounded wye low voltage network. The system serves a total load of around $250 \mathrm{kVA}$, figure 4 . The network integrates both single-phase as 3-phase loads.

\section{Simulation results}

The impact in network power losses value with different placement of DG is shown in figure 5 and 6.

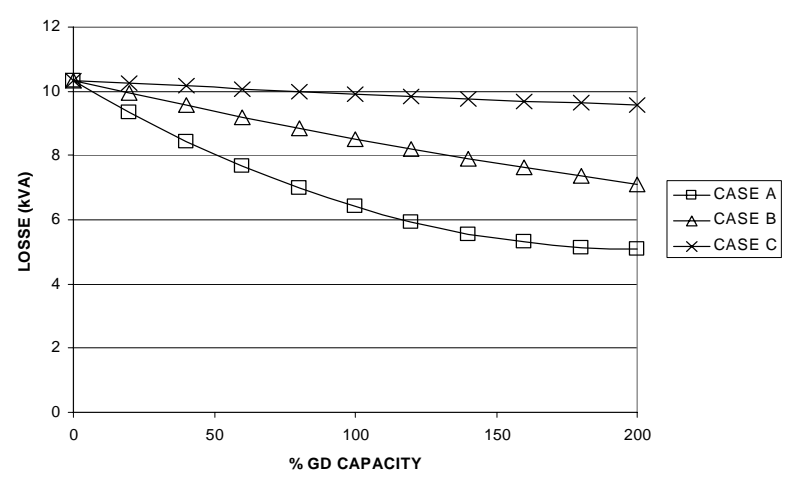

Fig. 5. Network Power Losses

The first scenario, CASE A, integrates a 3-phase DG unit in node 24. The second scenario, CASE B, integrates three single-phase DG units in node 26, 27 and 28. The last simulated scenario, CASE C, integrates three singlephase DG units in node 3, 4 and 9. In all cases the DG units' penetration level vary from $0 \%$ to $200 \%$. The results shown that the power losses reduction is around $50 \%$ in case A, $31 \%$ in case B and only $7 \%$ in case C. None of the cases exceeded the voltage limits (EN50160 - $\mathrm{U}_{\mathrm{n}} \pm 10 \%$ ).

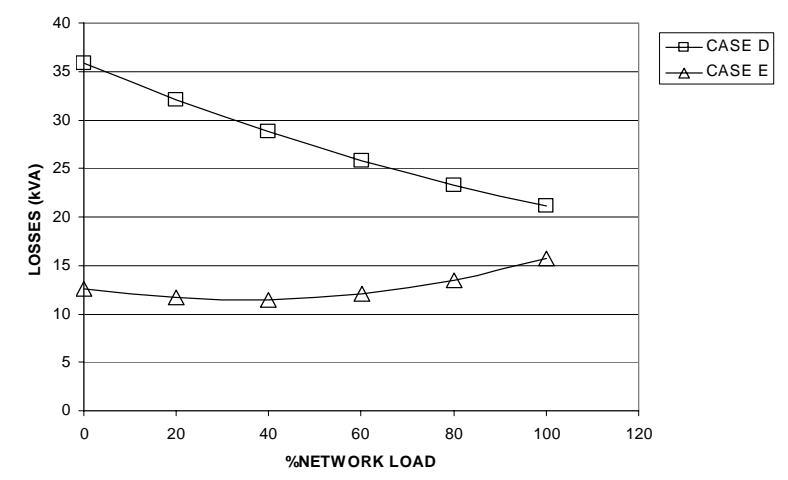

Fig. 6. Network Power Losses

The case D integrates 3-phase DG units in node 24. The case $\mathrm{E}$ integrates $\mathrm{DG}$ units in nodes 3,4 and 9 simultaneously. In both cases DG units have a fixed generation capacity of $50 \%$ of full network load. The rest of nodes vary from full load to minimum load. The results shown that the network power losses are higher in case D that in case E besides, case D, exceeded voltage limits imposed by the EN50160.
These basics analysis confirms the real benefit of DG integration into low voltage networks from the point of view of network power losses reduction. Furthermore results display the high importance of correct placement of DG in distribution network to avoid voltage limits violations. In order to optimize DG integration in low voltage distribution networks complex search techniques could be applied, like genetic algorithms or tabu search [13].

\section{Conclusions and further works}

A tool has been developed to test the impact of the integration of distributed generation on low voltage distribution networks. The 3-phase analysis capacity allows generation units with 3-phase, 2-phase or single phase connection to be integrated. The speed the power flow algorithm can be run allows the incorporation of dynamic model of loads and DG units with fast variation of demand or generation ratios.

The developed tool could possibly be improved in several ways. The actual tool is limited to grounded wye network. It could be possible to generalize to ungrounded-delta networks.

Future developments of the tool will enable control functions integration for centralised management of loads and distributed generation units. Functions like Active Demand Management and DG's voltage regulation management or optimisation of DG's production will be implemented running in conjunction with the dynamic power flow block.

\section{References}

[1] Manuel Sanchéz Jiménez, "Smart Electricity Networks based on large integration of Renewable sources and Distributed Generation", $\mathrm{PhD}$ thesis, University of Kassel, June 2006.

[2] RD 661/2007, 25th of May, by which special regime electrical energy generation activity is regulated.

[3] R. D. Zimmerman, H.D. Chiang, "Fast Decoupled Power Flow for Unbalanced Radial distribution Systems", IEEE Transactions on Power Systems, Vol. 10, No. 4, November, 1995, pp 2045-2052

[4] Carol S. Cheng, Dariush Shirmohammadi, "A threephase power flow method for real-time distribution analysis", IEEE Transactions on Power systems, Vol. 10, $\mathrm{N}^{0} 2$, May 1995, pp. 671-679.

[5] Ray Daniel Zimmerman, "Comprehensive distribution Power flow: modelling, formulation, solution Algorithm and analysis", Partial Fulfillment of the requirements for the Degree of Doctor of Philosophy, January 1995.

[6] A. Augugliaro, L. Dusonchet, S. Favuzza, M.G. Ippolito, E. Riva Sanseverino, "A compensation-based 
method to model PV nodes in backward/forward distribution network analysis", The International Journal for Computation and Mathematics in Electrical and Electronic Engineering, Vol. 26, № 2, 2007, pp 476-488

[7] A. Augugliaro, L. Dusonchet, M.G. Ippolito, S. Mangione, E. Riva Sanaeverino, "A modified backward/forward method for fast solving radial distribution networks", PowerTech Conference, Vol. 2, June, 2003, Bologna

[8] D. Rajicic, A. Dimitrovski, "A New Method for Handling PV Nodes in Backward/Forward Power flow for Radial and Weakly Meshed Networks", IEEE Porto Power Tech Conference, September 2001, Portugal

[9] D.Zimmerman, C. E. Murillo, D. Gan, MATPOWER 3.0, PSERC Cornell University, February 2005

[10] H.M. Mok, S. Elangovan, M.M.A. Salama, Cao Longijan, "Power Flow Analysis for Balanced and Unbalanced Radial Distribution Systems", Australian Universities Power Engineering Conference, Darwin, September 1999.

[11] IEEE Task Force on Load Representation for Dynamic Performance, Standard Load Models for Power Flow and Dynamic Performance Simulation, IEEE Transactions on Power Systems, Vol. 10, $\mathrm{N}^{\circ} 3$, August 1995.

[12] Norberto Redondo Melchor*, Roberto Carlos Redondo Melchor**, Ma Margarita Redondo Melchor**, "Cálculo de instalaciones ramificadas en baja tensión", Montajes e instalaciones September 2004.

[13] A. Robin Wallace, Gareth P.Harrison, "Planning for optimal accommodation of dispersed generation distribution networks", Proccedings $17^{\text {th }}$ International Conference on Electricity Distribution CIRED 2003, Barcelona, Spain, 12-15 May 2003.

\section{Annexe 1}

\begin{tabular}{|c|c|c|}
\hline Node & $P(k W)$ & Q (kVAr) \\
\hline 1 & & \\
\hline 2 & & \\
\hline $3-S$ & 5,75 & 1,9 \\
\hline $4-T$ & 5,75 & 1,9 \\
\hline 5 & & \\
\hline $6-T$ & 9,2 & 3,02 \\
\hline $7-T$ & 9,2 & 3,02 \\
\hline $8-S$ & 5,75 & 1,9 \\
\hline $9-R$ & 5,75 & 1,9 \\
\hline $10-R$ & 14,49 & 4,76 \\
\hline $11-S$ & 5,75 & 1,9 \\
\hline $12-S$ & 5,75 & 1,9 \\
\hline $13-S$ & 9,2 & 3,02 \\
\hline $14-R$ & 9,2 & 3,02 \\
\hline
\end{tabular}

\begin{tabular}{|c|c|c|}
\hline $15-T$ & 9,2 & 3,02 \\
\hline 16 & & \\
\hline $17-R S T$ & 20 & 6,57 \\
\hline $18-S$ & 14,49 & 4,76 \\
\hline $19-T$ & 5,75 & 1,9 \\
\hline $20-R$ & 5,75 & 1,9 \\
\hline 21 & & \\
\hline $22-T$ & 9,2 & 3,02 \\
\hline $23-R S T$ & 20 & 6,57 \\
\hline $24-R S T$ & 17 & 5,58 \\
\hline $25-R$ & 9,2 & 3,02 \\
\hline $26-R$ & 5,75 & 1,9 \\
\hline $27-S$ & 5,75 & 1,9 \\
\hline $28-T$ & 5,75 & 1,9 \\
\hline $29-S$ & 9,2 & 3,02 \\
\hline $30-R$ & 9,2 & 3,02 \\
\hline
\end{tabular}

